\title{
Transciptomic and histological analysis of hepatopancreas, muscle and gill tissues of oriental river prawn (Macrobrachium nipponense) in response to chronic hypoxia
}

\author{
Shengming Sun ${ }^{1}$, Fujun Xuan², Hongtuo Fu' ${ }^{1 *}$, Jian Zhu ${ }^{1 *}$, Xianping $\mathrm{Ge}^{1}$ and Zhimin $\mathrm{Gu}^{3}$
}

\begin{abstract}
Background: Oriental river prawn, Macrobrachium nipponense, is a commercially important species found in brackish and fresh waters throughout China. Chronic hypoxia is a major physiological challenge for prawns in culture, and the hepatopancreas, muscle and gill tissues play important roles in adaptive processes. However, the effects of dissolved oxygen availability on gene expression and physiological functions of those tissues of prawns are unknown. Adaptation to hypoxia is a complex process, to help us understand stress-sensing mechanism and ultimately permit selection for hypoxia- tolerant prawns, we performed transcriptomic analysis of juvenile M. nipponense hepatopancreas, gill and muscle tissues by RNA-Seq.
\end{abstract}

Results: Approximately 46,472,741; 52,773,612 and 58,195,908 raw sequence reads were generated from hepatopancreas, muscle and gill tissues, respectively. A total of 62,722 unigenes were generated, of the assembled unigenes, we identified 8,892 genes that were significantly up-regulated, while 5,760 genes were significantly down-regulated in response to chronic hypoxia. Genes from well known functional categories and signaling pathways associated with stress responses and adaptation to extreme environments were significantly enriched, including genes in the functional categories "response to stimulus", "transferase activity" and "oxidoreductase activity", and the signaling pathways "oxidative phosphorylation", "glycolysis/gluconeogenesis" and "MAPK signaling". The expression patterns of 18 DEGs involved in hypoxic regulation of M. nipponense were validated by quantitative real-time reverse-transcriptase polymerase chain reactions ( $(\mathrm{RT} T-P C R$; average correlation coefficient $=0.94$ ). In addition, the hepatopancreas and gills exhibited histological differences between hypoxia and normoxia groups. These structural alterations could affect the vital physiological functions of prawns in response to chronic hypoxia, which could adversely affect growth and survival of $M$. nipponense.

Conclusions: Gene expression changes in tissues from the oriental river prawn provide a preliminary basis to better understand the molecular responses of M. nipponense to chronic hypoxia. The differentially expressed genes (DEGs) identified in M. nipponense under hypoxia stress may be important for future genetic improvement of cultivated prawns or other crustaceans through transgenic approaches aimed at increasing hypoxia tolerance.

Keywords: Macrobrachium nipponense, RNA-Seq, Gene expression, Hypoxia

\footnotetext{
* Correspondence: fuht@ffrc.cn; zhuj@ffrc.cn

'Key Laboratory of Genetic Breeding and Aquaculture Biology of Freshwater Fishes, Ministry of Agriculture, Freshwater Fisheries Research Center, Chinese Academy of Fishery Sciences, Wuxi 214081, People's Republic of China Full list of author information is available at the end of the article
} 


\section{Background}

In aquatic environments, oxygen levels often fluctuate and crustaceans experience hypoxic conditions on a regular basis [1]. Macrobrachium nipponense, which is widely distributed in freshwater and low-salinity regions of estuaries, is one of the most important aquaculture species in China [2]. This freshwater prawn is an attractive model to study responses to hypoxia because it is relatively susceptible to hypoxic conditions compared to other crustaceans [3]. Hypoxic conditions cause stress that can inhibit the optimal development of crustaceans, resulting in reduced frequency of molts, metabolic changes, avoidance behavior, slow growth, suppression of immune function and even death [4-9]; however, few genetic markers to evaluate population genetics and perform phylogenic analysis [10-12] has meant that the molecular basis of chronic hypoxia exposure in Macrobrachium spp. is not well characterized. Recently, high-throughput transcriptomic sequencing was performed on the Illumina platform and this provided a genomic basis for further investigation of the hypoxia adaptation mechanisms of $M$. nipponense [13]. Breeding programs also eagerly desire a better understanding of physiological and genetic mechanisms underlying sensitivity to oxygen limitation.

Comparative studies between organisms inhabiting distinct environments can provide insight into the mechanisms that underlie responses to environmental differences [14]. In some cases, artificial treatments are used to create these differences in conditions and to facilitate comparisons [15-17]. To better understand the physiological and genetic changes in $M$. nipponense in response to hypoxia, comparative analysis between normoxic and hypoxic conditions is necessary. Previous research in M. nipponense has focused on the effects of hypoxia on respiratory metabolism [18], and there is growing evidence that changes in molecular indicators, such as energy production, antioxidant enzymes and oxygen-carrying proteins, can effectively imply chronic and acute hypoxia exposure in invertebrates [19-23]. When facing hypoxia, one of the main challenges of any tissue is to maintain energy levels either by increasing anaerobic energy-production, improving energyutilization or by lowering energy consumption; however, cellular adaptations to chronic hypoxia in hepatopancreas, gill and muscle tissues in prawns remain unexplained. As a result of this knowledge gap, large-scale expressed sequence tag (EST) libraries of M. nipponense have been sequenced [24-26], as these should allow more comprehensive molecular studies. Recently, high-throughput RNA-sequencing (RNA-Seq) has emerged as a strategy for analyzing the functional complexity of transcriptomes [13, 27, 28]. With the emergence of next generation sequencing, RNA-Seq is a relatively new technology allowing transcriptomic studies of the whole genome. Compared to traditional cDNA microarrays, RNA-Seq provides deep sequencing data for direct quantification of transcripts, which makes it more sensitive for detecting all expressed genes without the need for EST collection, probe synthesis, microarray design and hybridization.

In this present study, we used RNA-Seq to investigate genome-wide gene expression differences in M. nipponense in response to chronic hypoxia. To provide insight into the mechanisms underlying hypoxia-sensitivity in M. nipponense, it is necessary to identify hypoxia-related genes such as those in the hepatopancreas, muscle and gill tissues, as these are the most important organs for metabolism of nutrients, energy storage and immune responses, respectively. Our study highlights the pathways that react in response to chronic hypoxia through gene ontology (GO) and pathway analyses. This present study provides useful information to better understand the genetic-level responses of $M$. nipponense when exposed to chronic hypoxia.

\section{Results and discussion RNA-Seq data processing, reference assembly and alignment}

To provide comprehensive understanding of expression differences between $M$. nipponense cultured in normoxic and hypoxic conditions, we collected and deep sequenced RNA samples from hepatopancreas, muscle and gill tissues. A total of 308,348,065 paired-end reads were generated from six samples with 101-bp read length. The number of sequences in each sample ranged from 45.6 to 58.2 million (Table 1). After removal of ambiguous nucleotides, low-quality sequences (Phred quality scores $<20$ ), contaminated microbial sequences and ribosomal RNA sequences, a total of $265,294,865$ cleaned reads (86 \%) were harvested for further analysis. The cleaned sequences in each sample ranged from 31.5 to 52.7 million reads, thus confirming the stability and consistency of sampling, library preparation and sequencing methodologies. Using the Trinity assembly program, we generated a total of 62,722 unigenes, the average length was $960 \mathrm{bp}$, and the N50 length was 1,450 bp. (Table 2). The length distribution of unigenes is shown in Fig. 1. The cleaned reads of the six samples were pooled and assembled by the Trinity assembler to generate a reference transcriptome assoiated with published EST datas [29]. According to Gene Ontology (GO), an internationally standardized gene functional classification system, 11,104 nonredundant unigenes were classified into three major functional categories (biological process, cellular component and molecular function).

\section{Identification of DEGs}

We detected 13,466 DEGs between hypoxia and normoxia conditions in the hepatopancreas, muscle and gill tissues (false discovery rate $\leq 0.01$ and fold-change $\geq 2$; 
Table 1 Summary of sequence data generated for the Macrobrachium nipponense transcriptome, and quality filtering

\begin{tabular}{llllll}
\hline Group & Tissue & Reads & Clean reads & Total Clean Nucleotides (nt) & Q20 percentage \\
\hline Normoxia & Hepatopancreas & $46,472,741$ & $32,323,574$ & $4,865,587,148$ & $98.56 \%$ \\
& Muscle & $52,773,612$ & $50,213,656$ & $4,822,295,055$ & $98.42 \%$ \\
& Gill & $58,195,908$ & $52,684,676$ & $4,741,620,840$ & $98.26 \%$ \\
Hypoxia & Hepatopancreas & $45,590,728$ & $31,457,005$ & $4,731,627,788$ & $98.35 \%$ \\
& Muscle & $49,727,598$ & $47,295,194$ & $4,712,350,852$ & $98.15 \%$ \\
& Gill & $55,587,478$ & $51,320,754$ & $4,618,867,860$ & $97.78 \%$ \\
\hline
\end{tabular}

Fig. 2). Scatter plots were generated for FPKM values from the two treatment groups (Fig. 3). Of these DEGs, 4153, 1985 and 8814 genes were expressed in response to normoxia in hepatopancreas, muscle and gill tissues respectively (Additional file $1 \mathrm{~A}-\mathrm{C}$ ). A Venn diagram of the DEGs illustrates that the majority of these genes were not shared amongst the three tissue types, suggesting that the mechanisms and pathways employed in response to chronic hypoxia stress differ significantly between hepatopancreas, gill and muscle tissues (Fig. 4). A key regulator of cellular adaptations to hypoxia is the transcriptional activator hypoxia inducible factor 1 (HIF-1) [30]; however, in our present study with $M$. nipponense - and in previous studies on other invertebrates -HIF- $1 \alpha$ mRNA levels in the hepatopancreas consistently showed no response to hypoxia or anoxia [20,31-34]. The results suggest a model of hypoxia-induced regulation of HIF-1 $\alpha$ transcript abundance in crustaceans that may differs from that of typical HIF- $1 \alpha$ transcriptional regulation in mammalian systems. Since HIF exerts its action at the level of protein and not mRNA expression level, the absence of HIF among the DEG is not surprising.

\section{Functional analysis of DEGs in the hepatopancreas}

The metabolic oxygen demands of the hepatopancreas tissues in crustaceans are relatively high and similar across different species [35]. In response to chronic hypoxia, we observed significant gene transcription changes in the hepatopancreas under several GO terms, including upregulated genes for "oxidoreductase activity (GO:0016491)", "oxidation-reduction process (GO:0055114)" and "catalytic activity (GO:0003824)" and down-regulated genes "electron carrier activity (GO:0009055)", "immune system process (GO:0002376)" and "ion binding (GO:0043167)”

Table 2 Assembly statistics of reads

\begin{tabular}{ll}
\hline Parameter & Numbers \\
\hline Number of Unigene & 62,722 \\
Total bases of Unigene (bp) & $36,589,624$ \\
Unigene mean lengths (bp) & 960 \\
Minimum length (bp) & 300 \\
Max length (bp) & 26,388 \\
N50 length & 1450 \\
\hline
\end{tabular}

(Table 3). The genes in the molecular function category of "oxidoreductase activity" and "electron carrier activity" are widely studied and known to be associated with oxidative stress, mitochondrial function and adaptation to environmental stress. Moreover, expression profiling studies on other crustaceans have shown that a cluster of genes involved with oxidoreductase activity are differentially expressed in response to environmental stresses, such as temperature and confinement [21, 22, 36, 37]. Genes in the category "electron carrier activity" are responsible for extracting energy, and the slowing down of the respiratory chain caused by a lower oxygen concentration is consistent with reports on stress adaptation and resistance observed for many organisms. For instance, amino acid and ion transmembrane transporters are essential factors for hypoxia responses in many plants [38] and aquatic animals, including mollusks [39] and teleosts [40]. At same time, the mitochondrial electron transport chain is a major site of reactive oxygen species (ROS) production and hypoxic stress could increase hemocyte ROS production, probably because it partially inhibits mitochondrial electron transport producing redox changes in the electron carriers that increase ROS production at complex III $[41,42]$. The delicate balance between antioxidant defense and ROS production can be disrupted by compromised antioxidant defense or inhibition of electron flow [43], thus leaving $M$. nipponense potentially vulnerable to ROS damage during hypoxia.

\section{Functional analysis on DEGs in muscle tissue}

In response to chronic hypoxia, in muscle tissues we observed significant enrichment of genes for several GO terms related to stress responses. These GO terms included "catalytic activity (GO:0003824)" and "transcription regulator activity (GO:0030528)" in upregulated genes, and "chitin metabolic process (GO:0006030)" in the down-regulated genes. Since muscle is the main tissue of protein deposition and energy storage, we identified four glycolytic enzyme DEGs in $M$. nipponense in response to hypoxia, suggesting that these were important regulators in response to hypoxia stress in muscles. Under such hypoxic circumstances, the major source of energy is the glycolytic pathway with pyruvate as the substrate, while fermentative metabolism produces lactate 


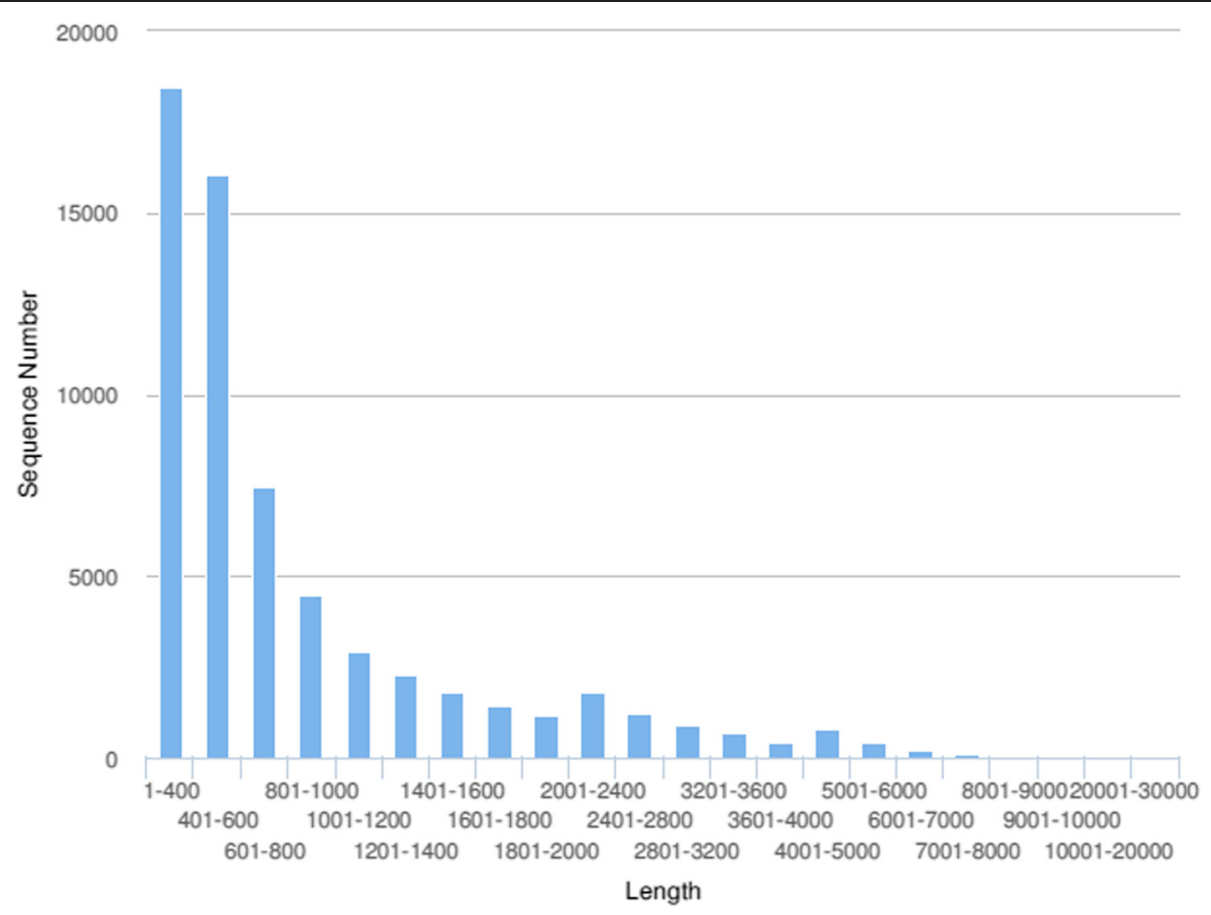

Fig. 1 Length distribution of assembled transcriptome unigenes from Macrobrachium nipponense. The x-axis indicates contig size and the $y$-axis indicates the number of unigenes of each size

Table $3 \mathrm{GO}$ enrichment analysis of genes up- or down-regulated in response to hypoxia stress

\begin{tabular}{|c|c|c|c|c|c|}
\hline Tissue & GO term & Definition & No. up- or down- regulated & Total in category & $P$-value \\
\hline \multirow[t]{8}{*}{ Hepatopancreas } & Up & & & & \\
\hline & GO:0016491 & Oxidoreductase activity & 58 & 773 & $8.20 \mathrm{E}-09$ \\
\hline & GO:0003824 & Catalytic activity & 33 & 2720 & $4.50 \mathrm{E}-08$ \\
\hline & GO:0055114 & Oxidation-reduction process & 62 & 551 & $5.21 \mathrm{E}-08$ \\
\hline & Down & & & & \\
\hline & GO:0002376 & Immune system process & 57 & 1697 & $4.58 \mathrm{E}-08$ \\
\hline & GO:0022904 & Respiratory electron transport chain & 47 & 119 & $8.09 \mathrm{E}-08$ \\
\hline & GO:0043167 & lon binding & 78 & 2466 & $5.43 \mathrm{E}-08$ \\
\hline \multirow[t]{6}{*}{ Muscle } & Up & & & & \\
\hline & GO:0003824 & Catalytic activity & 104 & 2720 & 7.99E-08 \\
\hline & GO:0045182 & Transcription regulator activity & 113 & 2466 & $6.77 \mathrm{E}-06$ \\
\hline & Down & & & & \\
\hline & GO:0006030 & Chitin metabolic process & 11 & 58 & 0.007 \\
\hline & GO:0006936 & Muscle contraction & 4 & 31 & 0.011 \\
\hline \multirow[t]{7}{*}{ Gill } & Up & & & & \\
\hline & GO:0016901 & Oxidoreductase activity & 32 & 523 & 0.004 \\
\hline & GO:0007154 & Cell communication & 28 & 152 & $2.68 \mathrm{E}-07$ \\
\hline & Down & & & & \\
\hline & GO:0050896 & Response to stimulus & 15 & 373 & $8.66 \mathrm{E}-08$ \\
\hline & GO:0042221 & Response to chemical stimulus & 30 & 251 & $3.81 \mathrm{E}-06$ \\
\hline & GO:0002376 & Immune system process & 28 & 75 & 3.07E-07 \\
\hline
\end{tabular}


Table 4 List of DEGs in hepatopancreas, muscle and gill tissues of pranws in response chronic hypoxia

\begin{tabular}{|c|c|c|c|c|}
\hline \multirow{2}{*}{$\frac{\text { Genes }}{\text { caspase-3 }}$} & \multirow{2}{*}{$\frac{\text { Gene ID }}{\text { isotig20204 }}$} & \multicolumn{3}{|c|}{ Differential expression tissues } \\
\hline & & & & gill \\
\hline inhibitor of apoptosis & isotig00582 & hepatopancreas & muscle & \\
\hline extracellular copper/zinc superoxide dismutase & isotig04224 & hepatopancreas & muscle & gill \\
\hline glutathione peroxidase & isotig15120 & hepatopancreas & & gill \\
\hline glutathione S-transferase & isotig17220 & hepatopancreas & muscle & gill \\
\hline peroxidase & isotig02186 & & muscle & \\
\hline phosphoenolpyruvate carboxykinase & isotig03978 & & muscle & \\
\hline hexokinase & isotig07588 & & muscle & \\
\hline pyruvate kinase & isotig03788 & & muscle & \\
\hline lactate dehydrogenase & isotig03261 & & muscle & \\
\hline arginine kinase 2 & isotig02307 & hepatopancreas & muscle & \\
\hline $\mathrm{Na}^{+} / \mathrm{K}^{+}$ATPase & isotig02174 & & & gill \\
\hline cathepsin L & isotig35781 & hepatopancreas & muscle & \\
\hline tropomyosin-2 & isotig00694 & & muscle & \\
\hline tubulin & isotig05597 & & muscle & \\
\hline myosin & isotig11489 & hepatopancreas & muscle & \\
\hline hemocyanin & isotig34775 & hepatopancreas & & \\
\hline carbonic anhydrase I & isotig02566 & hepatopancreas & & \\
\hline cytochrome c oxidase subunit I & isotig00782 & hepatopancreas & muscle & gill \\
\hline cytochrome oxidase subunit I & isotig05039 & hepatopancreas & & gill \\
\hline NADH dehydrogenase subunit 1 & isotig04226 & & & gill \\
\hline NADH dehydrogenase subunit 4 & isotig02071 & hepatopancreas & & gill \\
\hline ATP synthase & isotig00758 & hepatopancreas & & \\
\hline heat shock protein 70 & isotig27058 & & muscle & \\
\hline heat shock protein 90 & isotig04367 & hepatopancreas & muscle & \\
\hline heat shock protein 21 & isotig12922 & & muscle & \\
\hline heat shock protein 60 & isotig03937 & & & gill \\
\hline fatty acid synthase & isotig08686 & & & gill \\
\hline prophenoloxidase & isotig00567 & & & gill \\
\hline serine proteinase-like protein & isotig03292 & & muscle & \\
\hline plus agglutinin & isotig06982 & & & gill \\
\hline akirin & isotig02975 & & muscle & gill \\
\hline C-type lectin & isotig11437 & hepatopancreas & muscle & gill \\
\hline antimicrobial peptide & isotig29146 & & & gill \\
\hline alpha-2-Macroglobulins & isotig00388 & & & gill \\
\hline$\beta-1,3$-glucan binding protein & isotig26537 & & & gill \\
\hline
\end{tabular}

via lactate dehydrogenase (LD) [44]. Similar to previous studies [45-47], the primary effects of hypoxia on skeletal muscle were strong increases in the transcription of hexokinase (HK), pyruvate kinase (PK) and LD, which may be a precursor for increased glycolytic ATP production. However, lactate is toxic for the cells and leads to arrest of cellular functions and cell death during hypoxia. Besides its effect on cellular ATP, hypoxia can also influence regulation of the cytoskeleton as reflected by changes in tubulin and myosin proteins. Regulation of myosin by hypoxia has also been shown in the heart muscle of rats [48]. Tubulin- $\alpha$ and $-\beta$ form the microtubules that are responsible for cellular functions including mitosis, cell motility and shape $[49,50]$.

\section{Functional analysis on DEGs in gill tissue}

Crustacean gills make an important contribution to immune defenses and have well established functions in gaseous exchange and ion regulation. In response to 
Table 5 KEGG Pathway enrichment analysis of differentially expressed genes in response to hypoxia stress (P-value $<0.05)$

\begin{tabular}{lllllll}
\hline KEGG Pathway & Hepatopancreas & & Muscle & & Gill \\
& Number of genes & q-value & Number of genes & q-value & Number of genes & q-value \\
\hline Oxidative phosphorylation & 31 & 0.000 & 8 & 0.782 & 42 & 0.047 \\
MAPK signaling pathway & 10 & 0.000 & 9 & 0.006 & 9 & 0.028 \\
Lysosome & 26 & 0.014 & 8 & 0.306 & 36 & 0.056 \\
Glycolysis / gluconeogenesis & 28 & 0.000 & 12 & 0.006 & 15 & 0.183 \\
Fatty acid metabolism & 33 & 0.012 & 5 & 0.818 & 11 & 11 \\
Citrate cycle (TCA cycle) & 6 & 0.009 & 6 & 0.029 & 0.000 \\
Starch and sucrose metabolism & 17 & 0.000 & 3 & 0.194 & 13 & 0.292 \\
Insulin signaling pathway & 24 & 0.013 & 7 & 0.025 & 17 & 0.048 \\
Peroxisome & 24 & 0.000 & 2 & 0.000 & 12 & 0.975 \\
p53 signaling pathway & 4 & 0.602 & 7 & 0.013 & 9 & 0.045 \\
\hline
\end{tabular}

chronic hypoxia, in gill tissues we observed significant gene enrichment of several GO terms related to stress responses. These GO terms included "oxidoreductase activity (GO:0016901)" and "cell communication (GO:0007154)" in the upregulated genes, and "response to stimulus (GO:0050896)" and "response to chemical stimulus (GO:0042221)" in the down-regulated genes. Notably, there were 15 DEGs in the category "response to stimulus". We further investigated these DEGs and observed that heat shock protein (HSP) and glutathione S-transferase (GST) genes were down-regulated in the gills in response to hypoxia. HSPs target damaged proteins to the proteasome, which prevents the accumulation of dysfunctional proteins and permits the recycling of peptides and amino acids. This result suggests a high level of autophagy in the gills during hypoxia stress. One of the well-studied transferase

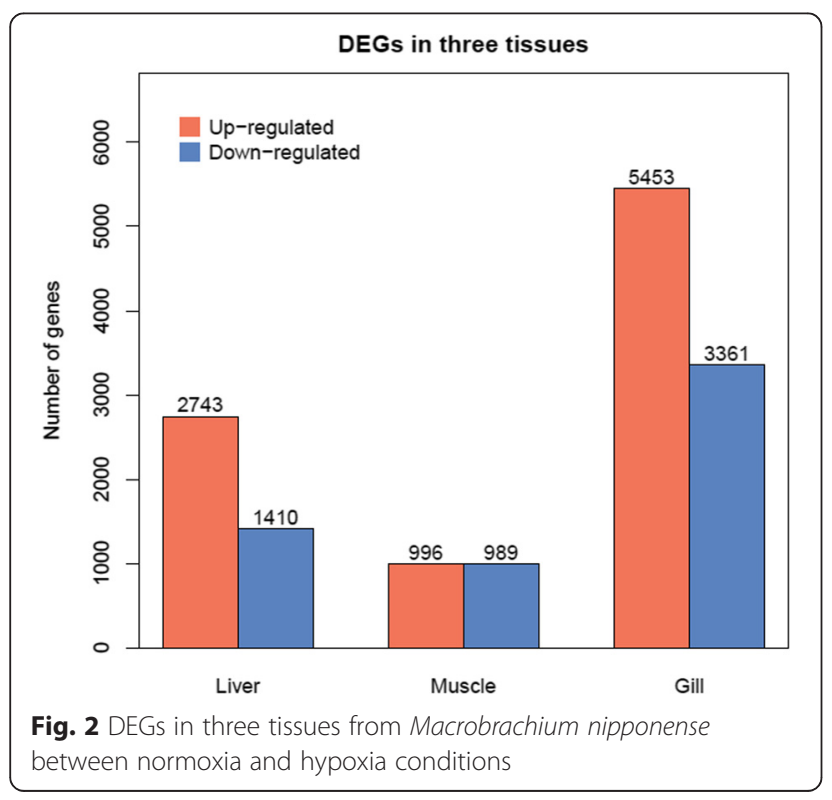

families is the GST gene family, as these genes perform essential functions in protecting cells against oxidative stress caused by various stresses, including toxic heavy metal ions [51-53], osmotic imbalance [54], salinity $[55,56]$ and $\mathrm{pH}$ changes [57]. GSTs have been used as biomarkers for environmental pollution and toxin detection $[58,59]$. We identified five DEGs belonging to the subcategory "immune system process" and there was down-regulation of prophenoloxidase (proPO), C-type lectin (CTL), antimicrobial peptide (AP) and alpha -2-macroglobulins $(\alpha 2 \mathrm{M})$, which are all important for disease resistance in crustaceans [60-63]. Therefore, prolonged hypoxia may have population consequences, as individuals that have already down-regulated their aerobic metabolism may also have decreased immune defenses, which could result in higher risk of outbreaking disease of prawn population.

\section{Confirmation of DEG candidate genes by real-time} reverse-transcriptase polymerase chain reaction (qRT-PCR) To validate the RNA-Seq results, 18 genes showing a high level of significance or known to play an important role in stress response functions were selected for qRTPCR analysis with $\beta$-actin acting as the reference gene (Table 4). No significant differences were shown between qRT-PCR and the Illumina data (Pearson's correlation coefficient $r=0.94$ ) (Fig. 5). In addition, a number of detoxification-related genes, such as GST and HSP, were also found to be specifically expressed in different tissues of $M$. nipponense in response to chronic hypoxia. These genes have been shown to be hypoxia-responsive in other studies [12, 21, 22], and they are involved in antioxidant abilities, immune responses, glycolysis and apoptosis, which are important functions for maintaining and re-establishing homeostasis in response to pathological changes. We also found that many genes related to mitochondrial respiration were significantly down-regulated under hypoxia stress 

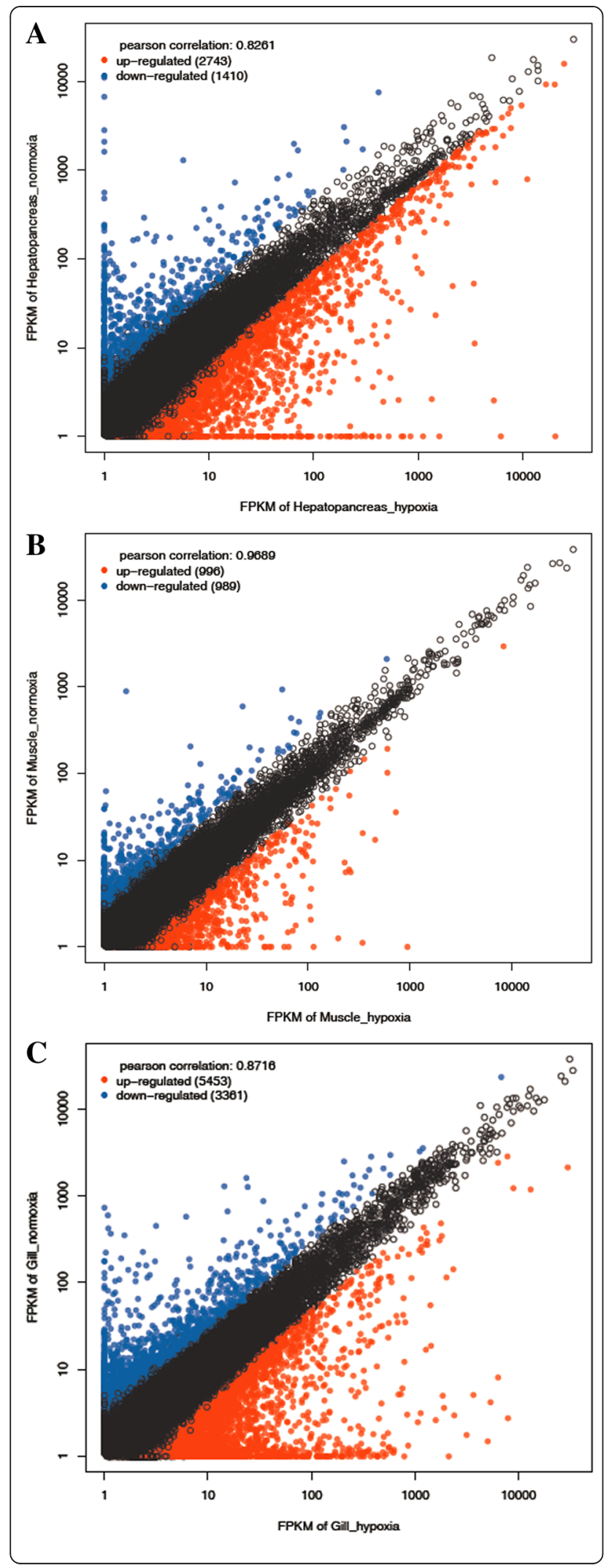

Fig. 3 Scatter-plot showing gene expression in three tissues from Macrobrachium nipponense. Average FPKM values for each gene in the gill $\mathbf{a}$, hepatopancreas $\mathbf{b}$ and muscle $\mathbf{c}$ tissues in hypoxia conditions correlated to average FPKM values for each gene in gill $\mathbf{a}$, hepatopancreas $\mathbf{b}$ and muscle $\mathbf{c}$ tissues in normoxia conditions

according to qRT-PCR, such as cytochrome c oxidase subunit I (CCO I), cytochrome oxidase I (COX I), NADH dehydrogenase subunit 1 (Complex I) and carbonic anhydrase I (CA I), which reveals that normal mitochondrial function was disrupted by oxidative stress. As mitochondrial respiration is affected in many pathologic conditions such as hypoxia and intoxications, the impaired electron transport chain could emit additional p53-inducing signals and thereby contribute to tissue damage [64]. We identified glycolysis related-genes from the DEG list for the muscle, and proPO, CTL, $\beta$-1,3-glucan binding protein (LGBP) and $\alpha 2 \mathrm{M}$ genes from the DEG list for the gill. Obviously, several genes could potentially be used as molecular indicators of hypoxia in $M$. nipponense at specific time points. However, the changes in the expression of these significant genes were too dynamic to serve as biomarkers of hypoxia stress in $M$. nipponense.

\section{KEGG pathway enrichment analysis}

Enrichment analysis is an effective way to identify the KEGG pathways that frequently occur in a tissue when the whole body transcriptome is used as the background $[65,66]$. Additional pathway mapping can facilitate the interpretation of significant gene data derived from complex biological processes and systems, especially when trying to characterize DEGs related to environmental toxicants or stressors [67]. Of the significantly expressed genes, $32 \%$ had a match in KEGG pathways targeted by chronic hypoxia. The most abundant categories were associated with oxidative phosphorylation, the MAPK signaling pathway, glycolysis/gluconeogenesis and citrate (TCA) cycle (Table 5). The major biological processes occurring in the mitochondria (TCA cycle, coupling electron transfer and oxidative phosphorylation) were altered remarkably in the hepatopancreas, muscle and gill tissues of M. nipponense. These results are in agreement with studies that have reported higher activities of glycolytic enzymes and fatty acid beta-oxidation process after exposure to an acute stressor in fish $[68,69]$, this may be necessary to cope with the increased energy demand of the tissues. The concentration of mitochondrial oxidative phosphorylation is tuned to the maximum energy conversion requirements of a given tissue, and it is hypothesized that its activity is modulated by tissue metabolic stress to maintain energy-metabolism homeostasis [70]. 

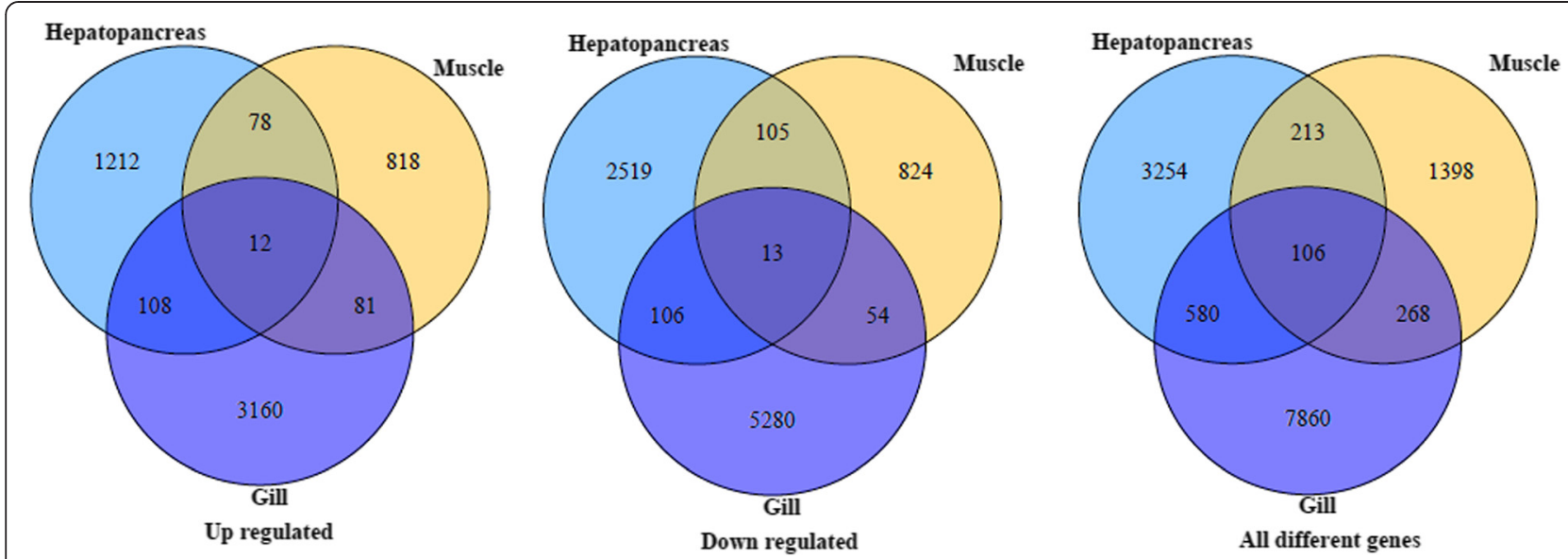

Fig. 4 Venn diagram of DEGs among three tissues from Macrobrachium nipponense

Histological studies of the hepatopancreas and gills of $M$. nipponense in response to hypoxia

The hepatopancreas is composed of numerous blind-end tubules, with each tubule consisting of different epithelial cell types, i.e. E-cell (embryonic), R-cell (resorptive), F-cell (fibrillar) and B-cell (blisterlike) [71]. Histological analysis of the hepatopancreas has been used as a practical means for assessing the environmental stress in the shrimp culture [72-74]. The R-cells in hepatopancreas epithelia are known to function as the main site for lipid storage [74]. There were distinct differences in the condition of the hepatopancreas in $M$. nipponense from normoxia and hypoxia groups (Fig. 6a \& b). The most obvious abnormalities were the hypertrophy of B-cells, and vacuoles that tended to coalesce into larger ones. Also, the number of Bcells was increased under hypoxia. The R-cells were compressed, appeared cuboidal and were reduced in number. The tubule lumens were mis-shaped and were enlarged due to a thinned epithelium. In crustaceans, the hepatopancreas is used for monitoring culture health and it serves as a sensitive indicator for metabolism, nutritional status and diseases in various shrimp species because it is the site of digestion, nutrient absorption, reserve storage, and synthesis and secretion of digestive enzymes [75, 76].

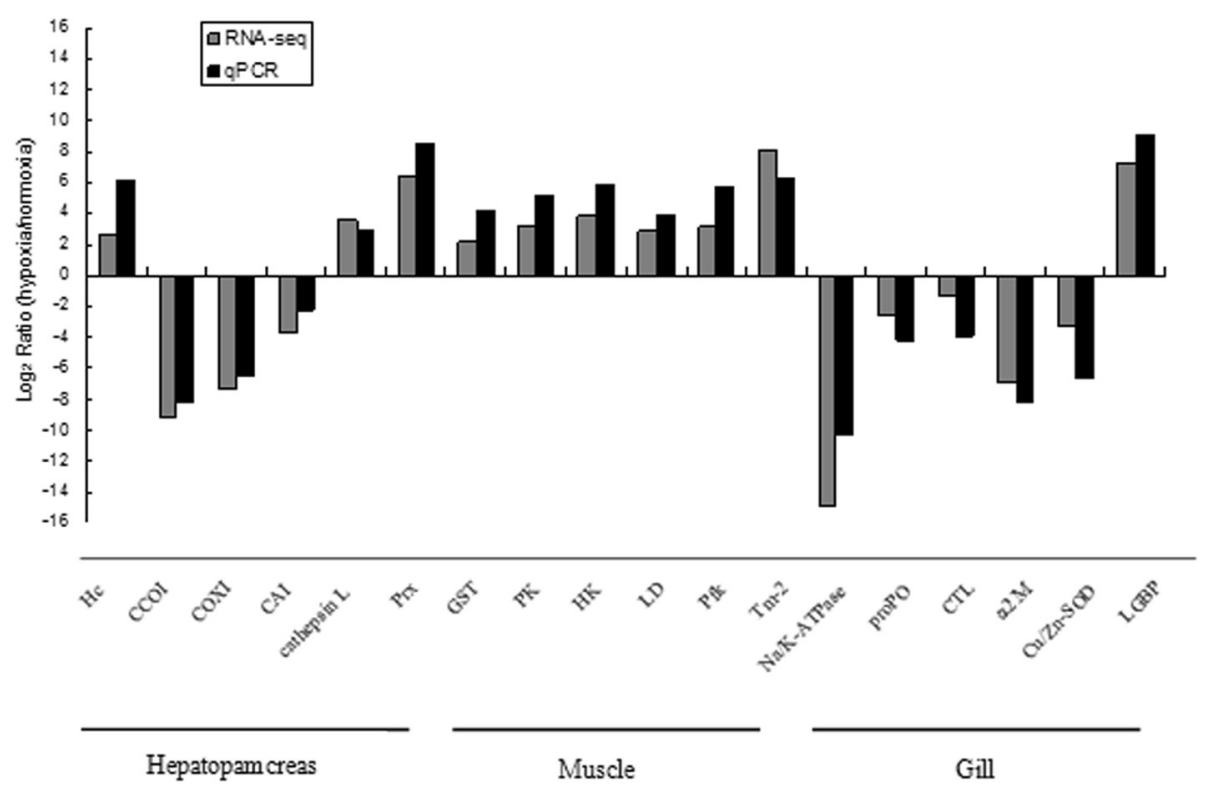

Fig. 5 Differentially expressed genes validated by qRT-PCR. Comparison between RNA-Seq results and qRT-PCR validation results. The x-axis shows genes in the three tissues validated in this study; the $y$-axis shows $\log _{2}$ Ratio of expression in Macrobrachium nipponense in response to hypoxia versus normoxia conditions 

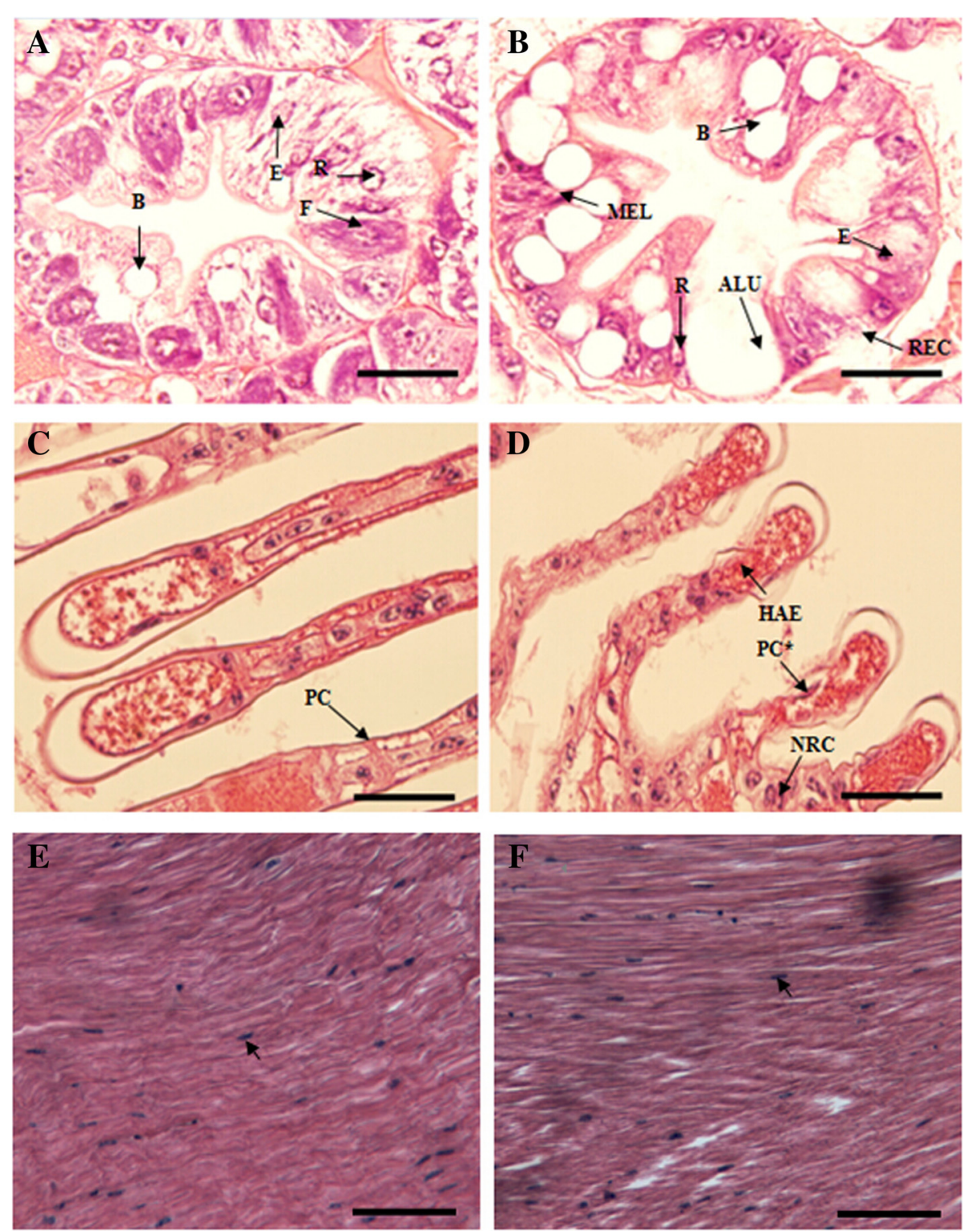

Fig. 6 Histopathological changes in the hepatopancreas, gill and muscle. Hepatopancreas of the Macrobrachium nipponense following a 7-day exposure to hypoxia: (a) control prawns; (b) prawns exposed to hypoxia at $2.5 \mathrm{mg} / \mathrm{L}$ dissolved oxygen, showing the appearance of large numbers of vacuoles in the tubular epithelial cells and abnormal lumens. ALU, abnormal lumen; MEL, melanization of cells; REC, ruptured epithelial cells. Anterior gill lamellae of the M. nipponense following a 7-day exposure to hypoxia: (c) control prawns showing normal lamellae structure and intact pillar cells (PC); (d) prawns exposed to hypoxia at $2.5 \mathrm{mg} / \mathrm{L}$ dissolved oxygen showing disrupted pillar cells (PC*), necrosis (NCR) and extensive infiltration of haemocytes (HAE). Histological cross sections of muscle of (e) control group and (f) prawns exposed to hypoxia at $2.5 \mathrm{mg} / \mathrm{L}$ dissolved oxygen showing simialr striated muscle fibers infiltrated by hemocytes (small arrowhead). Scale bars $=50 \mu \mathrm{m}$

Our study has also captured indirect evidence that oxidative stress suppressed antioxidant gene expression and disrupted fatty acid metabolism due to the observation of vacuolation in R-cells. The histoarchitecture of gills in the hypoxia group showed hemocytic infiltration, fused gill lamellae, mild malformations, swollen and necrotic lamellae, and complete disorganization of lamellae (Fig. 6c \& d). All these incidents can eventually lead to gill injury, which leads to disturbance in oxygen consumption [77] and enzymatic activities [78] in aquatic organisms. However, no obvious alterations were observed by light microscopy in the muscle tissues of $M$. nipponense after chronic hypoxia (Fig. 6e \& f).

\section{Conclusions}

We performed comparative transcriptome profiling of M. nipponense in response to hypoxia, and identified a relatively large number of genes that displayed distinct 
differences in expression in hepatopancreas, muscle and gill tissues. Further analysis revealed that several well-known functional categories of genes and signaling pathways associated with stress responses and energy metabolism were significantly enriched, including genes in the functional categories "response to stimulus", "transferase activity" and "oxidoreductase activity", and signaling pathways of "oxidative phosphorylation", "glycolysis/gluconeogenesis" and "TCA cycle". Overall, our transcriptome map may provide significant insights into the underlying molecular mechanisms that govern the responses of $M$. nipponense to hypoxia stress. The hypoxia-induced and -reduced genes identified may prove to be potential candidates for global genetic engineering of salt tolerance in M. nipponense. The pathways, the gene expression patterns and histological variations in gills and hepatopancreas tissues provide insight into understanding the molecular mechanisms of hypoxia in crustaceans.

\section{Methods}

\section{Ethics statement}

This study was approved by the Animal Care and Use Committee of the Centre for Applied Aquatic Genomics at Chinese Academy of Fishery Sciences.

\section{Prawn sampling}

Several healthy oriental river prawns with wet weights of 2.86-3.45 g were obtained from Tai Lake in Wuxi, China $\left(120^{\circ} 13^{\prime} 44^{\prime \prime} \mathrm{E}, 31^{\circ} 28^{\prime} 22^{\prime \prime} \mathrm{N}\right)$. All the samples were transferred to the laboratory of the Freshwater Fisheries Research Center and maintained in six 300-L tanks with aerated freshwater for one week prior to experimentation. During acclimation periods, prawns were hand-fed a commercial feed (Tongwei Group Co., Ltd.) containing 41.0 \% crude protein at $5 \%$ wet body weight twice daily. The culture conditions were $22.6 \pm 0.5^{\circ} \mathrm{C}, \mathrm{pH} 8.2 \pm 0.08$, dissolved oxygen $6.5 \pm 0.2 \mathrm{mg} / \mathrm{L}$, total ammonia-nitrogen 0.08 $0.09 \mathrm{mg} / \mathrm{L}$, and a photoperiod of $14 \mathrm{~h}$ light $/ 10 \mathrm{~h}$ dark.

The control group was maintained under normoxic conditions $(6.5 \pm 0.2 \mathrm{mg} \mathrm{O} / 2)$. Hypoxic $(2.5 \pm 0.1 \mathrm{mg} / \mathrm{L}$ dissolved oxygen) conditions in the treatment tanks were maintained for 7 days by bubbling with $\mathrm{N}_{2}$ gas until the desired $\mathrm{O}_{2}$ concentrations were reached; oxygen levels were maintained by adding $\mathrm{N}_{2}$ gas when needed. Oxygen concentrations were measured daily using a YSI 55oxygen meter (Yellow Springs, OH, USA). All exposures were conducted in triplicate for control and experimental group with three tanks in each group. After 7 days, the hepatopancreas, muscle and gill tissues of six prawns under hypoxia were removed as pooling samples in each tank, respectively. In parallel, six prawns held in air-saturated water (normoxia) were pooled as each negative control tank. Finally, these samples were immediately frozen in liquid nitrogen and stored at $-80{ }^{\circ} \mathrm{C}$ until being processed. The study was approved by the Institutional Animal Care and Use Ethics Committee of Freshwater Fisheries Research Center, Chinese Academy of Fishery Sciences (Wuxi, China).

\section{RNA extraction and quality control}

Total RNA was extracted from each tissue using TRIZOL (Invitrogen, Carlsbad, CA, USA) according to the manufacturer's instructions. RNA samples were digested by DNase I to eliminate potential genomic DNA. Integrity and size distribution were checked on a Bioanalyzer 2100 with RNA 6000 Nano Labchips (Agilent technologies, Santa Clara, CA, USA). Equal amounts of the high quality RNA sample from each tissue were then pooled for RNA-Seq. cDNA library construction and sequencing and RNA-Seq library preparation and sequencing were carried out as previously described [79]. cDNA libraries were prepared with $2.5 \mu \mathrm{g}$ total RNA following the protocols of the Illumina TruSeq RNA Sample Preparation Kit (Illumina). After KAPA quantitation and dilution, the library was sequenced on an Illumina HiSeq 2000 with 100-bp paired-end reads. After removing adaptor sequences, ambiguous ' $\mathrm{N}$ ' nucleotides (with the ratio of ' $\mathrm{N}$ ' greater than $5 \%$ ) and low quality sequences (with quality score less than 10 ), the remaining clean reads were assembled using trinity software [29] as described for de novo transcriptome assembly without a reference genome and this generated the reference sequences including a number of EST sequences available in public domains for the comparative transcriptome study.

\section{Functional annotation of assembled contigs}

The assembled transcriptome contigs were subjected to similarity searches against the NCBI non-redundant (nr) protein database using BLASTx with e-value cutoff of 1e-10. Gene name and description was assigned to each contig based on the BLASTx hit with the highest score. $\mathrm{GO}$ analysis was conducted on the assembled transcriptome using InterProScan (http://www.ebi.ac.uk/Tools/pfa/ iprscan/) and integrated protein databases with default parameters. The GO terms associated with transcriptome contigs were then obtained for describing their biological processes, molecular functions and cellular components. For pathway enrichment analysis, all DEGs were mapped to terms in the KEGG database and searched for significantly enriched KEGG terms compared to the whole transcriptome background. Functional enrichment analysis, including GO and KEGG, were performed using the ultrageometric test to identify which DEGs were significantly enriched in GO terms (P-value $\leq 0.05$ ) and metabolic pathways (q-value $\leq 0.05$ ) compared with the whole transcriptome background [80, 81]. 


\section{Read mapping and DEG analysis}

All the cleaned reads were mapped to the assembled reference transcriptome by Bowtie [28], and about $70 \%$ of the reads could be mapped to the reference for each sample (Table 1). RSEM was then used to estimate and quantify gene and isoform abundances according to the Trinity-assembled transcriptome. Finally, we used edgeR to normalize the expression levels in each of these samples and obtain the differentially expressed transcripts by pairwise comparisons [82]. EdgeR uses a negative binomial distribution method for differential expression analysis.

\section{qRT-PCR}

qRT-PCR was used to validate the expression of 18 DEGs. The sequences of the primer pairs (designed using Primer Express 3.0) are listed in Additional file 2. qRT-PCR reactions were carried out using the Bio-Rad iCycler iQ5 Real Time System (Biorad Inc., Berkeley, CA, USA) using the $\beta$-actin gene as internal control [83]. The PCR temperature profile and reaction conditions were performed according to the instructions of the SYBR Premix Ex Taq kit (TaKaRa, Dalian, China). For the negative control, diethypyrocarbonate (DEPC)water replaced the template. A relative standard curve was constructed using 10-fold serially diluted cDNA. Each sample was run in triplicate along with the internal control gene. To ensure that only one PCR product was amplified and detected, a dissociation curve analysis of amplification products was performed at the end of each PCR reaction. The relative copy number of gene mRNAs was calculated according to the $2^{-\Delta \Delta C T}$ comparative $C T$ method [84]. Pearson correlations between the qPCR data (average expression for each experimental group) and the Illumina data obtained by RNA-seq were calculated for each candidate gene.

\section{Histopathology}

Due to the hepatopancreas, gill and muscle of prawns being the main organs where multiple oxidative reactions, respiratory metabolism and genergy production occur with high metabolic activity. Three prawns from the hypoxia and normoxia groups were used to determine the histopathological effects of 7 days of hypoxia on the hepatopancreas, gill and muscle of prawns. The hepatopancreas, gill and muscle were removed and preserved in $10 \%$ neutral-buffered formalin for 7 days. Then the samples were rinsed in $70 \%$ ethanol and stored until further processing. The samples were dehydrated in isopropanol, cleared in xylene, infiltrated in paraffin, and then sectioned to a thickness of $5 \mu \mathrm{m}$. Sections were stained with hematoxylin and eosin and examined with a light microscope (Nikon Phase Contrast 0.90 Dry Japan). Hepatopancreas, gill and muscle tissue morphology was assessed in order to observe possible alterations revealed through differences in the condition of cell morphology.

\section{Availability of supporting data}

Raw sequencing data is available through the NCBI Sequence Read Archive under Project Accession SRP056408 (http://www.ncbi.nlm.nih.gov/sra/?term=SRP056408).

\section{Additional files}

Additional file 1: DGE in three different tissues.

Additional file 2: Primers used in validation of DEGs.

\section{Competing interests}

The authors declare that they have no competing interests.

\section{Authors' contributions}

SS conceived the study, and drafted the manuscript. SS and FX worked on sample collection and RNA sequencing, and participated in major bioinformatic analysis and manuscript preparation. SS and HF worked on DEG pipeline development. HF and JZ participated in gene pathway analysis. ZG participated in sample collection. XG participated in discussions and provided advices. All authors read and approved the final manuscript.

\section{Acknowledgements}

We acknowledge Yan Wang (Shanghai Majorbio Biopharm Biotechnology Co., Ltd.) for their kind help in sequencing and bioinformatics analysis. This work was supported by National Natural Science Foundation of China (No. 31402280), by the Open Fund of Agriculture Ministry Key Laboratory of Healthy Freshwater Aquaculture of the Ministry of Agriculture (ZJK2013092), the National "Twelfth Five-Year" Plan for Science \& Technology Support (2012 BAD25B07), and the Science \& Technology Supporting Program of Jiangsu Province (Grant No. BE2012334).

\section{Author details}

${ }^{1}$ Key Laboratory of Genetic Breeding and Aquaculture Biology of Freshwater Fishes, Ministry of Agriculture, Freshwater Fisheries Research Center, Chinese Academy of Fishery Sciences, Wuxi 214081, People's Republic of China. ${ }^{2}$ Jiangsu Provincial Key Laboratory of Coastal Wetland Bioresources and Environmental Protection, Yancheng City, Jiangsu Province 224002, People's Republic of China. ${ }^{3}$ Agriculture Ministry Key Laboratory of Healthy Freshwater Aquaculture, Zhejiang Institute of Freshwater Fisheries, Huzhou 313001, People's Republic of China.

Received: 25 February 2015 Accepted: 15 June 2015

Published online: 03 July 2015

\section{References}

1. Cheng W, Liu CH, Kuo CM. Effects of dissolved oxygen on hemolymph parameters of freshwater giant prawn, Macrobrachium rosenbergii (de Man). Aquaculture. 2003;220:843-56.

2. Ma K, Feng J, Lin J, Li J. The complete mitochondrial genome of Macrobrachium nipponense. Gene. 2011;487:160-5.

3. Li ZH, Wang JX, Xie S. Functions of environmental factors in shrimp aquaculture. Reservoir Fisheries. 2004;24:1-4 (In Chinese).

4. Mangum CP. Adaptation of the oxygen transport system to hypoxia in the blue crab, Callinectes sapidus. Am Zool. 1997;37:604-11.

5. Rosas C, Martinez E, Gaxiola G, Brito R, Sánchez A, Soto LA. Effect of dissolved oxygen on the energy balance and survival of Penaeus setiferus juveniles. Mar Ecol Prog Ser. 1998;174:67.

6. McMahon BR. Respiratory and circulatory compensation to hypoxia in crustaceans. Resp Physiol. 2001;128:349-64.

7. Wu RS. Hypoxia: from molecular responses to ecosystem responses. Mar Pollut Bull. 2002;45:35-45. 
8. Racotta IS, Palacios E, Mendez L. Metabolic responses to short and long-term exposure to hypoxia in white shrimp (Penaeus vannamei). Mar Freshw Behav Phy. 2002;35:269-75.

9. Qiu RJ. Effect of hypoxia on immunological, physiological response, and hepatopancreatic metabolism of juvenile Chinese mitten crab Eriocheir sinensis. Aquacult Int. 2011;19:283-99.

10. Zhao Y, Wang H, Ji XS, Zeng YQ, Yang PP, Ding L. Isolation and characterization of 20 polymorphic microsatellite markers in Macrobrachium nipponense. Conserv Genet Resour. 2010;2:137-9.

11. Song KH, Kim W. Isolation and characterization of polymorphic microsatellites from the oriental river prawn Macrobrachium nipponense (Caridea: Palaemonidae). J Crustacean Biol. 2011;31:138-41.

12. Qiao H, Lv D, Jiang SF, Sun SM, Gong YS, Xiong YW, et al. Genetic diversity analysis of oriental river prawn, Macrobrachium nipponense, in Yellow River using microsatellite marker. Genet Mol Res. 2013;12:5694-703

13. Sun SM, Xuan FJ, Ge XP, Fu HT, Zhu J, Zhang SY. Identification of differentially expressed genes in hepatopancreas of oriental river prawn, Macrobrachium nipponense exposed to environmental hypoxia. Gene. 2014:534:298-306.

14. Xu J, Li Q, Xu LM, Wang SL, Jiang YL, Zhao ZX, et al. Gene expression changes leading extreme alkaline tolerance in Amur ide (Leuciscus waleckii) inhabiting soda lake. BMC Genomics. 2013;14:682.

15. Deng Y, Yao J, Wang X, Guo H, Duan D. Transcriptome Sequencing and Comparative Analysis of Saccharina japonica (Laminariales Phaeophyceae) under Blue Light Induction. PLoS ONE. 2012;7:e39704.

16. Huang QS, Dong S, Fang C, Wu X, Ye T, Lin Y. Deep sequencing-based transcriptome profiling analysis of Oryzias melastigma exposed to PFOS. Aquat Toxicol. 2012;120-121:54-8.

17. Smith $S$, Bernatchez $L$, Beheregaray LB. RNA-seq analysis reveals extensive transcriptional plasticity to temperature stress in a freshwater fish species. BMC Genomics. 2013;14:375.

18. Guan YQ, Li L, Wang HC, Wang ZL. Effects of hypoxiaon respiratory metabolism and antioxidant capability of Macrobrachium nipponense. J Hebei Uni. 2010;30(3):301-6 (in Chinese with English abstract).

19. Brouwer M, Larkin P, Brown-Peterson N, King C, Manning S, Denslow N. Effects of hypoxia on gene and protein expression in the blue crab, Callinectes sapidus. Mar Environ Res. 2004;58:787-92.

20. Brouwer M, Brown-Peterson NJ, Laikin P. Molecular and whole animal responses of grass shrimp, Palamonetes pugio, exposed to chronic hypoxia. J Exp Mar Biol Ecol. 2007;341:16-31

21. Li T, Brouwer M. Hypoxia inducible factor, gsHIF, of the grass shrimp Palaemonetes pugio: molecular characterization and response to hypoxia. Comp Biochem Physiol B Biochem Mol Biol. 2007;147:11-9.

22. Li TD, Brouwer M. Gene expression profile of grass shrimp Palaemonetes pugio exposed to chronic hypoxia. Comp Biochem Physiol D Genomics Proteomics. 2009;4:196-208.

23. Li TD, Brouwer M. Gene expression profile of hepatopancreas from grass shrimp Palaemonetes pugio exposed to cyclic hypoxia. Comp Biochem Physiol D Genomics Proteomics. 2013;8:1-10.

24. Wu P, Qi D, Chen L, Zhang H, Zhang X, Qin JG, et al. Gene discovery from an ovary CDNA library of oriental river prawn Macrobrachium nipponense by ESTs annotation. Comp Biochem Physiol D Genomics Proteomics. 2009:4:111-20

25. Qiao H, Fu H, Jin S, Wu Y, Jiang S, Gong Y, et al. Constructing and random sequencing analysis of normalized cDNA library of testis tissue from oriental river prawn (Macrobrachium nipponense). Comp Biochem Physiol D Genomics Proteomics. 2012;7(3):268-76.

26. Ma K, Qiu G, Feng J, Li J. Transcriptome Analysis of the Oriental River Prawn, Macrobrachium nipponense Using 454 Pyrosequencing for Discovery of Genes and Markers. Plos One. 2012;7(6):e39727.

27. Wang Z, Gerstein M, Snyder M. RNA-Seq: a revolutionary tool for transcriptomics. Nat Rev Genet. 2009;10:57-63.

28. Marioni JC, Mason CE, Mane SM, Stephens M, Gilad Y. RNA-seq an assessment of technical reproducibility and comparison with gene expression arrays. Genome Res. 2008;18:1509-17.

29. Grabherr MG, Haas BJ, Yassour M, Levin JZ, Thompson DA, Amit I, et al Full-length transcriptome assembly from RNA-Seq data without a reference genome. Nat Biotechnol. 2011;29(7):644-52.

30. Wenger RH, Stiehl DP: Camenisch G. Integration of oxygen signaling at the consensus HRE. Sci STKE. 2005;2005:re12.
31. Soñanez-Organis JG, Peregrino-Uriarte AB, Gómez-Jiménez S, López-Zavala A, Forman HJ, Yepiz-Plascencia G. Molecular characterization of hypoxia inducible factor-1 (HIF-1) from the white shrimp Litopenaeus vannamei and tissue specific expression under hypoxia. Comp Biochem Physiol C Pharmacol Toxicol. 2009;150:395-405

32. Ivanina AV, Sokolov EP, Sokolova IM. Effects of cadmium on anaerobic energy metabolism and mRNA expression during air exposure and recovery of an intertidal mollusk Crassostrea virginica. Aquat Toxicol.

2010;99:330-42

33. Piontkivska H, Chung JS, Ivanina AV, Sokolov EP, Techa S, Sokolova IM. Molecular characterization and mRNA expression of two key enzymes of hypoxia-sensing pathways in eastern oysters Crassostrea virginica (Gmelin): hypoxia-inducible factor a (HIF-a) and HIF-prolyl hydroxylase (PHD). Comp Biochem Physiol D Genomics Proteomics. 2011;6:103-14.

34. Susarellu R, Fabioux C, Sanchez MC, Le Goïc N, Lembert C, Soudant P, et al. Molecular and cellular response to short-term oxygen variations in the Pacific oyster Crassostrea gigas. J Exp Mar Biol Ecol. 2012;412:87-95.

35. Maciel FE, Rosa CE, Santos EA, Monserrat JM, Nery LEM. Daily variations in oxygen consumption, antioxidant defenses, and lipid peroxidation in the gills and hepatopancreas of an estuarine crab. Can J Zool. 2004:82:1871-7.

36. Brown-Peterson NJ, Larkin P, Denslow N, King C, Manning S, Brouwer M. Molecular indicators of hypoxia in the blue crab Callinectes sapidus. Mar Ecolo Prog Ser. 2005;286:203-15.

37. Brown-Peterson NJ, Manning CS, Patel V, Denslow ND, Brouwer M. Effects of cyclic hypoxia on gene expression and reproduction in a grass shrimp, Palaemonetes pugio. Bioligical Bulletin. 2008;214:6-16.

38. Rocha M, Licausi F, Araújo WL, Nunes-Nesi A, Sodek L, Fernie AR, et al. Glycolysis and the tricarboxylic acid cycle are linked by alanine aminotransferase during hypoxia induced by waterlogging of Lotus japonicus. Plant Physiology. 2010;152:1501-13.

39. Sussarellua R, Fabiouxa C, Le Moullacb G, Fleuryc E, Moragaa D. Transcriptomic response of the Pacific oyster Crassostrea gigas to hypoxia. Mar Genom. 2010;3:133-43.

40. Wulffa $T$, Jokumsen A, Hjrupc P, Jessen F. Time-dependent changes in protein expression in rainbow trout muscle following hypoxia. J proteomics. 2012;75:2342-51

41. Chandel NS, Maltepe E, Goldwasser E, Mathieu CE, Simon MC, Schumacker PT. Mitochondrial reactive oxygen species trigger hypoxia-induced transcription. Proc Natl Acad Sci USA. 1998;95:11715-20.

42. Chandel NS, McClintock DS, Feliciano CE, Wood TM, Melendez JA, Rodriguez AM, et al. Reactive oxygen species generated atmitochondrial complex III stabilize hypoxia-inducible factor1-a during hypoxia. J Biol Chem. 2000;275:25130-8

43. Turrens JF. Mitochondrial formation of reactive oxygen species. J Physiol London. 2003;552(2):335-44.

44. Tadege M, Dupuis I, Kuhlemeier C. Ethanolic fermentation: new functions for an old pathway. Trends Plant Sci. 1999;4:320-5.

45. Gracey AY, Troll Joshua V, Somero GN. Hypoxia-induced gene expression profiling in the euryoxic fish Gillichthys mirabilis. Proc Natl Acad Sci USA. 2001:98:1993-8.

46. Rees BB, Figueroa YG, Wiese TE, Beckman BS, Schulte PM. A novel hypoxiaresponse element in the lactate dehydrogenase-B gene of the killifish Fundulus heteroclitus. Comp Biochem Physiol A Mol Integr Physiol. 2009;154:707.

47. Koenig S, Solé M. Muscular cholinesterase and lactate dehydrogenase activities in deepsea fish from the NW Mediterranean. Mar Environ Res. 2014:94:16-23.

48. Razeghi P, Essop MF, Huss JM, Abbasi S, Manga N, Taegtmeyer H. Hypoxiainduced switches of myosin heavy chain iso-gene expression in rat heart. Biochem Biophys Res Commun. 2003;303:1024-7.

49. Frixione E. Recurring views on the structure and function of the cytoskeleton: a 300-year epic. Cell Motil Cytoskeleton. 2000;46:73-94.

50. Valiron O, Caudron N, Job D. Microtubule dynamics. Cell Mol Life Sci. 2001;58:2069-84

51. Yadav S. Heavy metals toxicity in plants: an overview on the role of glutathione and phytochelatins in heavy metal stress tolerance of plants. S Afr J Bot. 2010;76(2):167-79.

52. Espinoza HM, Williams CR, Gallagher EP. Effect of cadmium on glutathione S-transferase and metallothionein gene expression in coho salmon liver, gill and olfactory tissues. Aquat Toxicol. 2012;110:37-44. 
53. Srikanth K, Pereira E, Duarte A, Ahmad I. Glutathione and its dependent Enzymes' modulatory responses to toxic metals and metalloids in fish-a review. Environ Sci Pollut Res Int. 2013;20(4):2133-49.

54. Choi CY, An KW, An MI. Molecular characterization and mRNA expression of glutathione peroxidase and glutathione S-transferase during osmotic stress in olive flounder (Paralichthys olivaceus). Comp Biochem Physiol A Mol Integr Physiol. 2008;149(3):330-7.

55. Zanette J, de Almeida EA, da Silva AZ, Guzenski J, Ferreira JF, Di Mascio P, et al. Salinity influences glutathione S-transferase activity and lipid peroxidation responses in the Crassostrea gigas oyster exposed to diesel oil. Sci Total Environ. 2011;409(10):1976-83.

56. Li EC, Wang SL, Li C, Wang XD, Chen K, Chen LQ. Transcriptome sequencing revealed the genes and pathways involved in salinity stress of Chinese mitten crab, Eriocheir sinensis. Physiol Genomics. 2014;46:177-90.

57. Zhou J, Wang W-N, Wang A-L, He W-Y, Zhou Q-T, Liu Y, et al. Glutathion S-transferase in the white shrimp Litopenaeus vannamei: Characterization and regulation under $\mathrm{pH}$ stress. Comp Biochem Physiol C Toxi Pharmacol. 2009;150(2):224-30.

58. Vidal-Liñán L, Bellas J. Practical procedures for selected biomarkers in mussels, Mytilus galloprovincialis-Implications for marine pollution monitoring. Sci Total Environ. 2013:461:56-64.

59. Fabrini R, Bocedi A, Del Grosso E, Morici L, Federici G, Palleschi A, et al. Erythrocyte glutathione transferase: A novel biomarker to check environmental pollution hazardous for humans. Biochem Biophys Res Commun. 2012;426(1):71-5

60. Gai YC, Zhao JM, Song LS, Lia CH, Zheng PL, Qiu LM, et al. A prophenoloxidase from the Chinese mitten crab Eriocheir sinensis: Gene cloning, expression and activity analysis. Fish Shellfish Immunol. 2008;24(2):156-67.

61. Xu YH, Bi WJ, Wang XW, Zhao YR, Zhao XF, Wang JX. Two novel C-type lectins with a low-density lipoprotein receptor class A domain have antiviral function in the shrimp Marsupenaeus japonicus. Dev Comp Immunol. 2014;24(2):323-32.

62. Hou FJ, He SL, Liu YJ, Zhu XX, Sun CG, Liu XL. RNAi knock-down of shrimp Litopenaeus vannamei Toll gene and immune deficiency gene reveals their difference in regulating antimicrobial peptides transcription. Dev Comp Immunol. 2014;44(2):255-60.

63. Shanthi S, Vaseeharan B. Alpha 2 macroglobulin gene and their expression in response to GFP tagged Vibrio parahaemolyticus and WSSV pathogens in Indian white shrimp Fenneropenaeus indicus. Aquaculture. 2014;418-419:48-54.

64. Khutornenko AA, Roudko W, Chernyak BV, Vartapetian AB, Chumakov PM, Evstafieva AG. Pyrimidine biosynthesis links mitochondrial respiration to the p53 pathway. Proc Natl Acad Sci USA. 2010;107(29):12828-33.

65. Mao X, Cai T, Olyarchuk JG, Wei L. Automated genome annotation and pathway identification using the KEGG Orthology $(\mathrm{KO})$ as a controlled vocabulary. Bioinformatics. 2005;21:3787-93.

66. Fukushima A, Kusano M, Redestig H, Arita M, Saito K. Metabolomic correlationnetwork modules in Arabidopsis based on a graph-clustering approach. BMC Syst Biol. 2011;5:1.

67. Currie RA, Bombail V, Oliver JD, Moore DJ, Lim FL, Gwilliam V, et al. Gene Ontology mapping as an unbiased method for identifying molecular pathways and processes affected by toxicant exposure: application to acute effects caused by the rodent non-genotoxic carcinogen diethylhexylphthalate. Toxicol Sci. 2005;86:453-69.

68. Du FK, Xu GC, Nie ZJ, Xu P, Gu RB. Transcriptome analysis gene expression in the liver of Coilia nasus during the stress response. BMC Genomics. 2014;15:558

69. Mininni AN, Milan M, Ferraresso S, Petochi T, Di Marco P, Marino G, et al. Liver transcriptome analysis in gilthead sea bream upon exposure to low temperature. BMC Genomics. 2014;15:765.

70. Phillips D, Covian R, Aponte AM, Glancy B, Taylor JF, Chess D, et al. Regulation of oxidative phosphorylation complex activity: effects of tissue-specific metabolic stress within an allometric series and acute changes in workload. Am J Physiol Regul Integr Comp Physiol. 2012;302:R1034-48.

71. Franceschini-Vicentini IB, Ribeiro K, Papa LP, Junior JM, Vicentini CA, Valent PMCM. Histoarchitectural features of the hepatopancreas of the Amazon River prawn Macrobrachium amazonicum. Int J Morphol. 2009;27:121-8.

72. Wu JP, Chen HC, Huang DJ. Histopathological and biochemical evidence of hepatopancreatic toxicity caused by cadmium and zinc in the white shrimp, Litopenaeus vannamei. Chemosphere. 2008;73:1019-26.

73. Stalina A, Broosa KV, Sadiq Bukharia A, Syed Mohamed HE, Singhalb RK, Venu-Babuca P. Morphological and histological studies on freshwater prawn
Macrobrachium rosenbergii (de man) irradiated with60Co gammaradiation. Aquat Toxicol. 2013;144-145:36-49.

74. Johnston DJ, Calvert KA, Crear BJ, Carter CG. Dietary carbohydrate/lipid ratios and nutritional condition in juvenile southern rock lobster, Jasus edwardsii. Aquaculture. 2003;220:667-82

75. Calvo NS, Stumpf L, Pietrokovsky S, López Greco LS. Early and late effects of feed restriction on survival, growth and hepatopancreas structure in juveniles of the red claw crayfish Cherax quadricarinatus. Aquaculture. 2011;319:355-62

76. Sousa LG, Petriella AM. Histology of the hepatopancreas of the freshwater prawn Palaemonetes argentinus (Crustacea: Caridea). Biocell. 2000;24:189-95.

77. Cebrian C, Andreu-Moliner ES, Fernandez-Casalderrey A, Ferrando MD. Acute toxicity and oxygen consumption in the gills of Procambarus clarkii inrelation to chlorpyrifos exposure. Bull Environ Contam Toxicol. 1992;49:145-9.

78. Chang CC, Lee PP, Hsu JP, Yeh SP, Cheng W. Survival, and bio-chemical, physiological, and histopathological responses of the giant freshwaterprawn, Macrobrachium rosenbergii, to short-term trichlorfon exposure. Aquaculture. 2006;253:653-66

79. Jin S, Fu H, Zhou Q, Sun S, Jiang S, Xiang Y, et al. Transcriptome Analysis of Androgenic Gland for Discovery of Novel Genes from the Oriental River Prawn, Macrobrachium nipponense, Using Illumina Hiseq 2000. PLoS ONE. 2013;8(10):e76840

80. Kanehisa M, Goto S, Hattori M, Aoki-Kinoshita KF, Itoh M, Kawashima S, et al. From genomics to chemical genomics: new developments in KEGG. Nucleic Acids Res. 2006;34:D354-7.

81. Kanehisa M, Araki M, Goto S, Hattori M, Hirakawa M, Itoh M, et al. KEGG for linking genomes to life and the environment. Nucleic Acids Res. 2008;36:D480-4.

82. Robinson MD, McCarthy DJ, Smyth GK. edgeR: a Bioconductor package for differential expression analysis of digital gene expression data. Bioinformatics. 2010;26(1):139-40.

83. Zhang YP, Fu HT, Qiao H, Jin SB, Jiang SF, Xiong YW, et al. Molecular cloning and expression analysis of transformer-2 gene during development in Macrobrachium nipponense (de Haan). J World Aquacult Soc. 2013;44:338-49.

84. Livak KJ, Schmittgen TD. Analysis of relative gene expression data using real-time quantitative PCR and the 2 (-Delta Delta C (T)) Method. Methods. 2012;5:402-8.

\section{Submit your next manuscript to BioMed Central and take full advantage of:}

- Convenient online submission

- Thorough peer review

- No space constraints or color figure charges

- Immediate publication on acceptance

- Inclusion in PubMed, CAS, Scopus and Google Scholar

- Research which is freely available for redistribution 